\title{
EXTREMAL LENGTH AND CONFORMAL CAPACITY $\left({ }^{1}\right)$
}

\author{
BY \\ WILLIAM P. ZIEMER( $\left.{ }^{2}\right)$
}

1. Introduction. The theory of extremal length has been successfully applied to conformal mappings, analytic functions of a complex variable, and in recent years it has found application in the study of quasiconformal mappings in space. Its application in quasiconformal mapping theory begins essentially with a theorem proved by Gehring [G3], that the conformal capacity of a space ring $R$ is directly related to the extremal length of the family of curves that join the boundary components of $R$. He has also shown that the conformal capacity is related to the extremal length of a family of surfaces that separate the boundary components of $R$. Gehring assumes that the separating surfaces are sufficiently smooth; Krivov [K] establishes a similar result under the assumption that the extremal metric is well-behaved. Under similar assumptions, other authors have dealt with the extremal length of separating surfaces, cf. [FU, Theorem 9], [SA], and [H].

The purpose of this paper is to eliminate the need for these assumptions. Thus, we consider the case of two disjoint compact sets $C_{0}, C_{1}$ contained in the closure of a bounded, open, connected set $G$. It is proved that the conformal capacity $C$ of $C_{0}, C_{1}$, relative to $G$ is related to the $n / n-1$-dimensional module $M$ of all closed sets that separate $C_{0}$ from $C_{1}$ in the closure of $G$ by

$$
C^{-1 /(n-1)}=M \text {. }
$$

This is accomplished by using a technique of Gehring's [G1, Lemma 3] which eliminates all assumptions concerning the behavior of the extremal metric. Then, a surface-theoretic approximation theorem, first developed in [FF, 8.23], permits the consideration of arbitrary closed separating sets.

The author wishes to thank George Springer, who suggested the problem under consideration in this paper and who has shown a continued interest in the development of its solution.

\section{Preliminaries.}

2.1. $E^{n}$ will denote Euclidean $n$-space and $L_{n}$ is $n$-dimensional Lebesgue measure. Hausdorff $k$-dimensional measure in $E^{n}$ will be denoted by $H^{k}$ (for its definition and properties see [F2]) and in this paper, only $H^{1}$ and $H^{n-1}$ will be used. If $A \subset E^{n}$, then bdry $A$ will mean the boundary of $A$ and for $x \in E^{n}, \delta(x, A)$ will be the distance from $x$ to $A$. More generally, $\delta(A, B)$ will denote the distance between

Presented to the Society, January 26, 1966; received by the editors May 24, 1966.

(') Abstract appears in Notices Amer. Math. Soc. 13 (1966), 323 (66T-206).

$\left({ }^{2}\right)$ This work was supported in part by a grant from the National Science Foundation. 
the sets $A$ and $B . S(x, r)$ will stand for the open $n$-ball of radius $r$ and centered at $x$. If $A$ is an $L_{n}$-measurable subset of $E^{n}$, then $\mathscr{L}^{p}(A)$ will denote those functions $f$ for which $|f|^{p}$ is $L_{n}$-integrable over $A$ and $\|f\|_{p}$ will be its $\mathscr{L}^{p}$ norm.

2.2. A real valued function $u$ defined on an open set $U \subset E^{n}$ is said to be absolutely continuous in the sense of Tonelli (ACT) on $U$ if it is ACT on every interval $I \subset U$, cf. [SS, p. 169]. Thus, the gradient of $u, \nabla u$, exists at $L_{n}$-almost every point in $U$. The following co-area formula, which was proved in [Z2], will be used frequently throughout this paper.

2.2.1. THEOREM. If $A$ is an $L_{n}$-measurable subset of $U$ and $u$ is $A C T$ on $U$, then

$$
\int_{A}|\nabla u(x)| d L_{n}(x)=\int_{-\infty}^{\infty} H^{n-1}\left[u^{-1}(s) \cap A\right] d L_{1}(s) .
$$

Therefore,

$$
\int_{U} f(x)|\nabla u(x)| d L_{n}(x)=\int_{-\infty}^{\infty} \int_{u^{-1}(s)} f(x) d H^{n-1}(x) d L_{1}(s)
$$

whenever $f \in \mathscr{L}^{1}(U)$.

2.3. Conformal capacity. The following will be standard notation throughout. $G$ is an open, bounded, connected set in $E^{n}$ and $C_{0}, C_{1}$, are disjoint compact sets in the closure of $G$. We will let $R=G-\left(C_{0} \cup C_{1}\right)$ and $R^{*}=R \cup C_{0} \cup C_{1}$. The conformal capacity of $C_{0}, C_{1}$ relative to the closure of $G$ is defined as

$$
C\left[G, C_{0}, C_{1}\right]=\inf \int_{R}|\nabla u|^{n} d L_{n}
$$

where the infimum is taken over all functions $u$ which are continuous on $R^{*}$, ACT on $R$, and assume boundary values 1 on $C_{1}$ and 0 on $C_{0}$. Such functions are called admissible for $C\left[G, C_{0}, C_{1}\right]$. Sometimes we will write $C$ for $C\left[G, C_{0}, C_{1}\right]$.

If $C_{0} \cup C_{1} \subset G$ and if $C_{0} \cup C_{1}$ consists of only a finite number of nondegenerate components, then the arguments of $[\mathrm{G} 2, \S \S 3-7]$ can be applied with only slight modifications to prove that the infimum in (1) is attained by a unique admissible function $u$ which is ACT in $G$. (By using the methods of Chapter III in [FU], one can prove the existence of an extremal for more general situations.) This extremal function $u$ satisfies the variational condition

$$
\int_{R}|\nabla u|^{n-2} \nabla u \cdot \nabla w d L_{n}=0
$$

for any function $w$ which is ACT on $G$, assumes boundary value 0 on $C_{0} \cup C_{1}$, and for which $|\nabla w| \in \mathscr{L}^{n}(R)$.

2.4. Integral currents. The following notion of exterior normal, which was first introduced in [F1, p. 48], will be used later. 
An $L_{n}$-measurable set $E \subset E^{n}$ has the unit vector $n(x)$ as exterior normal to $E$ at $x$ if, letting

we have

$$
\begin{aligned}
S(x, r) & =\{y:|y-x|<r\}, \\
S_{+}(x, r) & =S(x, r) \cap\{y:(y-x) \cdot n(x) \geqq 0\} \\
S_{-}(x, r) & =S(x, r) \cap\{y:(y-x) \cdot n(x) \leqq 0\} \\
\alpha & =L_{n}[S(x, 1)]
\end{aligned}
$$

$$
2 \lim _{r \rightarrow 0} L_{n}\left[S_{-}(x, r) \cap E\right] / \alpha r^{n}=1, \quad 2 \lim _{r \rightarrow 0} L_{n}\left[S_{+}(x, r) \cap E\right] / \alpha r^{n}=0 .
$$

The set of points $x$ for which $n(x)$ exists is called the reduced boundary of $E$ and will be denoted by $\beta(E)$. Obviously, $\beta(E) \subset$ bdry $E$. The importance of the exterior normal is seen in the following general version of the Gauss-Green Theorem [F3], [DG].

2.4.1. THEOREM. If $E$ is a bounded $L_{n}$-measurable set with $H^{n-1}[\beta(E)]<\infty$, then

$$
\int_{E} \operatorname{div} f(x) d L_{n}(x)=\int_{\beta(E)} f(x) \cdot n(x) d H^{n-1}(x)
$$

whenever $f: E^{n} \rightarrow E^{n}$ is continuously differentiable.

This theorem enables us to regard a bounded, open set $U \subset E^{n}$ with

$$
H^{n-1}(\text { bdry } U)<\infty
$$

as an integral current mod 2 (or integral class), i.e., an integral current $T$ with coefficients in the group of integers $\bmod 2,[\mathrm{Z1}, \S 3.6]$ or [FL]. Thus, if $\phi$ is a differential $n$-form of class $C^{\infty}$, then

$$
T(\phi)=\int_{U} \phi d L_{n}
$$

The boundary of $T, \partial T$, is defined as $\partial T(\omega)=T(d \omega)$ whenever $\omega$ is an $n-1$ form and $d \omega$ is its exterior derivative. Now $\beta(U)$ is a Hausdorff $(n-1)$-rectifiable set and therefore, 2.4.1 allows us to identify $\beta(U)$ with $\partial T,[\mathrm{Z1}, \S 3.6]$. Thus, the support of $\partial T$ is $\mathrm{cl}(\beta(U)) \subset$ bdry $U$, the mass of $T$ is $M(T)=L_{n}(U)$, and $M(\partial T)=H^{n-1}[\beta(U)]$ $[\mathrm{Z} 1, \S 3.6]$.

In view of this identification the following theorem is an immediate consequence of $[\mathrm{Z} 1,6.2]$ although the original version was given in [FF, 8.23]. An open set is called a convex cell if it can be expressed as the finite intersection of open halfspaces and an $n$-dimensional polyhedral set is the union of a finite number of convex cells.

2.4.2. THEOREM. If $U \subset E^{n}$ is a bounded, open set with $H^{n-1}($ bdry $U)<\infty$, then there is a sequence of $n$-dimensional polyhedral sets $P_{i}$ such that

(i) $P_{i} \subset\left\{x: \delta(x, U)<i^{-1}\right\}$,

(ii) $L_{n}\left(P_{i}\right) \rightarrow L_{n}(U)$ as $i \rightarrow \infty$,

(iii) $H^{n-1}$ (bdry $\left.P_{i}\right) \rightarrow H^{n-1}[\beta(U)]$ as $i \rightarrow \infty$. 
Moreover, by employing an argument similar to that of [FL, (5.6) and (5.7)], it can be arranged that

(iv) bdry $P_{i} \subset\left\{x: \delta[x, \beta(U)]<i^{-1}\right\}$.

Of course, one could apply [FL, 5.6 and p. 170] directly after verifying that the boundary of $U$ in Fleming's sense is $\beta(U)$. Finally, defining the measure $\mu_{i}$ as the restriction of $H^{n-1}$ to bdry $P_{i}$ and $\mu$ as the restriction of $H^{n-1}$ to $\beta(U)$, (iii) above and $[\mathrm{Z} 1,5.7]$ or $[\mathrm{FL}, 4.2]$ imply that

(v) $\mu_{i} \rightarrow \mu$ weakly as $i \rightarrow \infty$,

that is,

$$
\int f d \mu_{i} \rightarrow \int f d \mu \text { for every continuous } f .
$$

2.5. The module of a system of measures. Instead of dealing with extremal length, we prefer to work with the module as developed in [FU].

Let $\mathscr{M}$ be the class of all Borel measures on $E^{n}$. With an arbitrary system $E \subset \mathscr{M}$ of measures is associated a class of nonnegative Borel functions $f$ subject to the condition

$$
\int_{E^{n}} f d \mu \geqq 1 \quad \text { for every } \mu \in E .
$$

We will write $f \wedge \mu$ if (3) is satisfied and $f \wedge E$ if (3) is satisfied for every $\mu \in E$. For $0<p<\infty$, the module of $E, M_{p}(E)$, is defined as

$$
M_{p}(E)=\inf \left\{\int_{E^{n}} f^{p} d L_{n}: f \wedge E\right\}
$$

A statement concerning measures $\mu \in \mathscr{M}$ is said to hold for $M_{p}$-a.e. $\mu$ if the statement fails to hold for only a set of measures $E_{0}$ where $M_{p}\left(E_{0}\right)=0$. The proofs of the following statements can be found in [FU, Chapter 1].

(i) $M_{p}(E) \leqq M_{p}\left(E^{\prime}\right)$ if $E \subset E^{\prime}$,

(ii) $M_{p}(E) \leqq \sum_{i=1}^{\infty} M_{p}\left(E_{i}\right)$ if $E=\bigcup_{i=1}^{\infty} E_{i}$.

(iii) If $\bar{\mu}$ is the completion of $\mu$ and $L_{n}(A)=0$, then $\bar{\mu}(A)=0$ for $M_{p}$-a.e. $\mu \in \mathscr{M}$.

(iv) If $f \in \mathscr{L}^{p}\left(E^{n}\right)$, then $f$ is $\bar{\mu}$ integrable for $M_{p}$-a.e. $\mu \in \mathscr{M}$.

(v) If $\left\|f_{i}-f\right\|_{p} \rightarrow 0$, then there is a subsequence $f_{i}$, such that

$$
\int_{E^{n}}\left|f_{i,}-f\right| d \bar{\mu} \rightarrow 0 \quad \text { for } M_{p^{-a}} \text { a.e. } \mu \in \mathscr{M} .
$$

(vi) If $E \subset \mathscr{M}$, then $M_{p}(E)=0$ if and only if there is a nonnegative Borel function $f \in \mathscr{L}^{p}$ such that

$$
\int_{E^{n}} f d \mu=\infty \quad \text { for every } \mu \in E .
$$

(vii) If $p>1$ and $E \subset \mathscr{M}$, then there is a nonnegative Borel function $f$ such that

$$
\int_{E^{n}} f^{p} d L_{n}=M_{p}(E)
$$

and $\int f d \mu \geqq 1$ for $M_{p}$-a.e. $\mu \in E$. 
For the applications in this paper, the measures $\mu$ are complete (in fact, they are the restrictions of Hausdorff measure to compact sets) and for such measures, we have the following

2.5.1 THEOREM. If $p \geqq 2, E_{1} \subset E_{2} \subset \cdots$ are sets of complete measures, and $E=\bigcup E_{i}$, then

$$
M_{p}(E)=\lim _{i \rightarrow \infty} M_{p}\left(E_{i}\right) .
$$

Proof. In view of (i) above, observe that the limit exists and is dominated by $M_{p}(E)$. Therefore, only the case where the limit is finite needs to be considered.

For each $i$, let $f_{i}$ be the Borel function associated with $E_{i}$ as in (vii) above. Clarkson's inequality [C] states, for any $i$ and $j$,

(5) $\int_{E^{n}}\left|\frac{f_{i}+f_{j}}{2}\right|^{p} d L_{n}+\int_{E^{n}}\left|\frac{f_{i}-f_{j}}{2}\right|^{p} d L_{n} \leqq 2^{-1} \int_{E^{n}}\left|f_{i}\right|^{p} d L_{n}+2^{-1} \int_{E^{n}}\left|f_{j}\right|^{p} d L_{n}$.

If $i>j$, then $2^{-1} \cdot\left(f_{i}+f_{j}\right) \wedge \mu$ for $M_{p}$-a.e. $\mu \in E_{j}$. Therefore, because of (5),

$$
\int_{E^{n}}\left|\frac{f_{i}-f_{j}}{2}\right|^{p} d L_{n} \leqq 2^{-1} M_{p}\left(E_{i}\right)-2^{-1} M_{p}\left(E_{j}\right) .
$$

The right side of (6) tends to zero as $i, j \rightarrow \infty$ with $i>j$ and therefore, there is a nonnegative function $f$ such that $\left\|f_{i}-f\right\|_{p} \rightarrow 0$. Thus, from the above properties of module (especially (v)), we have that $f \wedge \mu$ for $M_{p}$-a.e. $\mu \in E$. This implies

$$
M^{p}(E) \leqq \int_{E^{n}} f^{p} d L_{n}=\lim _{i \rightarrow \infty} M_{p}\left(E_{i}\right)
$$

which is all that is required to prove.

3. Module of separating sets and conformal capacity. In this section we will establish the relationship between conformal capacity and arbitrary closed separating sets. $G, R^{*}, R, C_{1}$, and $C_{0}$ will be as defined in $\S 2.3$.

We will say that a set $\sigma \subset E^{n}$ separates $C_{0}$ from $C_{1}$ in $R^{*}$ if $\sigma \cap R$ is closed in $R$ and if there are disjoint sets $A$ and $B$ which are open in $R^{*}-\sigma$ such that $R^{*}-\sigma=A \cup B, C_{0} \subset A$, and $C_{1} \subset B$. Let $\Sigma$ denote the class of all sets that separate $C_{0}$ from $C_{1}$ in $R^{*}$. With every $\sigma \in \Sigma$, associate a complete measure $\mu$ in the following way: for every $H^{n-1}$-measurable set $A \subset E^{n}$, define

$$
\mu(A)=H^{n-1}(A \cap \sigma \cap R) .
$$

From the properties of Hausdorff measure, it is clear that the Borel sets of $E^{n}$ are $\mu$-measurable and therefore the module of $\Sigma$ can be as defined as in 2.5. Thus, for $n^{\prime}=n / n-1$,

$$
M_{n^{\prime}}(\Sigma)=\inf \left\{\int_{E^{n}} f^{n^{\prime}} d L_{n}: f \wedge \Sigma\right\}
$$

where $f \wedge \Sigma$ means that $f$ is a nonnegative Borel function on $E^{n}$ such that

$$
\int_{\sigma \cap R} f d H^{n-1} \geqq 1 \quad \text { for every } \sigma \in \Sigma \text {. }
$$


3.1. As in [V1, 2.3] it can be shown that if $\Sigma^{\prime}$ denotes those $\sigma \in \Sigma$ for which $H^{n-1}(\sigma \cap R)=\infty$, then $M_{n^{\prime}}\left(\Sigma^{\prime}\right)=0$.

3.2. Lemma. Let $u$ be an admissible function for $C\left[G, C_{0}, C_{1}\right]$ (see 2.3) and let $S \subset[0,1]$ be an $L_{1}$-measurable set. If $\Sigma(S)=\left\{u^{-1}(s): s \in S\right\}$ and $M_{n^{\prime}}[\Sigma(S)]=0$, then $L_{1}(S)=0$.

Proof. Since $M_{n^{\prime}}[\Sigma(S)]=0$, (vi) of 2.5 provides a nonnegative Borel function $f \in \mathscr{L}^{n^{\prime}}$ such that

$$
\int_{u^{-1}(s) \cap R} f d H^{n-1}=\infty \quad \text { for every } s \in S
$$

However, Hölder's inequality and the co-area formula (2.2.1) imply

$$
\infty>\int_{R} f|\nabla u| d L_{n} \geqq \int_{0}^{1} \int_{u^{-1}(s) \cap R} f(x) d H^{n-1}(x) d L_{1}(s)
$$

and therefore $L_{1}(S)=0$.

3.3. THEOREM. $M_{n^{\prime}}(\Sigma) \geqq C\left[G, C_{0}, C_{1}\right]^{-1 / n-1}$.

Proof. Choose $\varepsilon>0$ and let $f$ be any function for which $f \wedge \Sigma$. Let $u$ be an admissible function for $C=C\left[G, C_{0}, C_{1}\right]$ such that

$$
\int_{R}|\nabla u|^{n} d L_{n}<C+\varepsilon
$$

$R^{*}$ is connected since $G$ is and therefore it is clear that $u^{-1}(s) \in \Sigma$ for all $0<s<1$. Hence, by Hölder's inequality and 2.2.1, we have

$$
\begin{aligned}
\left(\int_{E^{n}} f^{n^{\prime}} d L_{n}\right)^{1 / n^{\prime}}(C+\varepsilon)^{1 / n} & \geqq\left(\int_{E^{n}} f^{n^{\prime}} d L_{n}\right)^{1 / n^{\prime}}\left(\int_{R}|\nabla u|^{n} d L_{n}\right)^{1 / n} \\
& \geqq \int_{R} f|\nabla u| d L_{n} \\
& \geqq \int_{0}^{1} \int_{u^{-1}{ }_{(s)}} f d H^{n-1}(x) d L_{1}(s) \geqq 1
\end{aligned}
$$

Since $\varepsilon$ is arbitrary,

$$
\int_{R} f^{n^{\prime}} d L_{n} \geqq C^{-1 / n-1}
$$

which is also true for the infimum over all $f \wedge \Sigma$, and thus the result follows.

3.4. The opposite inequality will be established by means of a sequence of approximations and we will begin by first assuming that $C_{0} \cup C_{1}$ consists only of a finite number of nondegenerate continua and that $C_{0} \cup C_{1} \subset G$. We will also assume initially that $H^{n-1}($ bdry $G)<\infty$. 
Let $V$ be an open connected set such that $G \supset \operatorname{cl} V \supset V \supset C_{0} \cup C_{1}$ and let $v$ be the extremal function for $C\left[V, C_{0}, C_{1}\right]$ (see 2.3). Since $v$ satisfies the variational condition (2), the proof of the following lemma is very similar to that of Lemma 3 in [G3] and will not be given here. It is possible to obtain a stronger result, but the following is sufficient for our purposes. Recall from 2.4 the definition of an $n$-dimensional polyhedral set.

3.5. LemMA. Let $\pi$ be the boundary of an n-dimensional polyhedral set $P$ ( $P$ not necessarily contained in $V$ ) such that $C_{0} \subset P$ and $C_{1} \subset E^{n}-\mathrm{cl} P$. Then $\pi$ separates $C_{0}$ from $C_{1}$ in $V$ and

$$
\int_{\pi(b)}|\nabla v|^{n-1} d L_{n} \geqq 2 b C\left[V, C_{0}, C_{1}\right]
$$

whenever $0<b<\delta\left(\pi, C_{0} \cup C_{1}\right)$ and where $\pi(b)=\{x: \delta(x, \pi)<b\}$.

3.6. REMARK. In Lemmas 3.7 and 3.8 , the integral average, $f_{r}$, of $|\nabla v|^{n-1}$ will be used. Thus, defining $\nabla v=0$ on the complement of $V$, for each $r>0$ we have

$$
f_{r}(x)=\alpha(r)^{-1} \int_{S(x, r)}|\nabla v(y)|^{n-1} d L_{n}(y)
$$

where $\alpha(r)=L_{n}[S(x, r)]$. It is well known that $f_{r}$ is continuous and that

$$
f_{r} \rightarrow|\nabla v|^{n-1} L_{n} \text {-a.e. } \quad \text { as } r \rightarrow 0 .
$$

Also, by a result of K. T. Smith [S] and Lebesgue's dominated convergence theorem, $\left\|f_{r}\right\|_{n^{\prime}} \rightarrow\left\||\nabla v|^{n-1}\right\|_{n^{\prime}}$ as $r \rightarrow 0$ and consequently, $\left\|f_{r}-|\nabla v|^{n-1}\right\|_{n^{\prime}} \rightarrow 0$.

3.7. LeMMA. With $\pi$ as in 3.5 ,

$$
\int_{\pi} f_{r} d H^{n-1} \geqq C\left[V, C_{0}, C_{1}\right]
$$

whenever $r<\delta\left(\pi, C_{0} \cup C_{1}\right)$.

Proof. Choose $b>0, r>0$ so that $b+r<\delta\left(\pi, C_{0} \cup C_{1}\right)$. If $\pi_{y}$ denotes the translation of $\pi$ through the vector $y$, then Fubini's Theorem and 3.5 imply

$$
\begin{aligned}
\int_{\pi(b)} f_{r}(x) d L_{n}(x) & =\alpha(r)^{-1} \int_{S(0, r)} \int_{\pi(b)}|\nabla v(x+y)|^{n-1} d L_{n}(x) d L_{n}(y) \\
& =\alpha(r)^{-1} \int_{S(0, r)} \int_{\pi_{y}(b)}|\nabla v(x)|^{n-1} d L_{n}(x) d L_{n}(y) \\
& \geqq 2 b C\left[V, C_{0}, C_{1}\right]
\end{aligned}
$$

since $\pi_{y}$ satisfies the conditions of 3.5. In addition to this, if $d(x)=\delta(x, \pi)$, then $|\nabla d|=1 L_{n}$-a.e. on $E^{n}-\pi[\mathrm{F} 4,(3)$ of 4.8$]$ and therefore 2.2 .1 gives

$$
\int_{\pi(b)} f_{r}(x) d L_{n}(x)=\int_{0}^{b} \int_{d^{-1}(s)} f_{r}(x) d H^{n-1}(x) d L_{1}(s) .
$$


Let $F(s)$ denote the inner integral on the right. Since $f_{r}$ is continuous on $E^{n}$ and $\pi$ is the boundary of a polyhedral set, it is clear that

$$
\lim _{s \rightarrow 0} F(s)=2 \int_{\pi} f_{r} d H^{n-1}
$$

Hence, from (7) and (8) we have

$$
C\left[V, C_{0}, C_{1}\right] \leqq \lim _{b \rightarrow 0}(2 b)^{-1} \int_{0}^{b} F(s) d L_{1}(s)=\int_{\pi} f_{r} d H^{n-1} .
$$

3.8. LeMMA. If $\Sigma$ is the class of sets that separate $C_{0}$ from $C_{1}$ in $G$, then

$$
\int_{\sigma \cap R}|\nabla v|^{n-1} d H^{n-1} \geqq C\left[V, C_{0}, C_{1}\right]
$$

for $M_{n^{\prime}}$-a.e. $\sigma \in \Sigma$.

Proof. Select $\sigma \in \Sigma$ and let $U$ be that part of a partition of $G-\sigma$ that contains $C_{0}$. Since $H^{n-1}$ (bdry $\left.G\right)<\infty$ by assumption, by appealing to 3.1 we can take $H^{n-1}$ (bdry $\left.U\right)<\infty$. Recall that $G \supset \operatorname{cl} V$ and that $\nabla v=0$ on the complement of $V$. Hence, we can choose $r_{0}$ so small that the support of $f_{r_{0}}$ is contained in $G$ (and therefore for all $r \leqq r_{0}$ ) and $r_{0}<\delta$ (bdry $U, C_{0} \cup C_{1}$ ).

By applying 2.4.2 to the set $U$, we obtain a sequence of $n$-dimensional polyhedral sets $P_{i}$. Let $\pi_{i}=$ bdry $P_{i}$. From properties (i), (ii), and (iv) of 2.4 .2 it is clear that eventually $C_{0} \subset P_{i}$ and $C_{1} \subset E^{n}-\operatorname{cl} P_{i}$. Thus Lemma 3.7 applies to $\pi_{i}$ for all large $i$. Now, $\beta(U) \subset$ bdry $U \subset$ (bdry $G) \cup \sigma$ and since the support of $f_{r}$ is contained in $G$ for all $r \leqq r_{0}$, it is clear that

$$
\int_{\sigma} f_{r} d H^{n-1} \geqq \int_{\beta(U)} f_{r} d H^{n-1} \quad \text { for all } r \leqq r_{0} .
$$

Since $f_{r}$ is continuous, (v) of 2.4 .2 and 3.7 imply

$$
\int_{\beta(U)} f_{r} d H^{n-1}=\lim _{i \rightarrow \infty} \int_{\pi_{i}} f_{r} d H^{n-1} \geqq C\left[V, C_{0}, C_{1}\right] \quad \text { for } r \leqq r_{0} .
$$

Thus, from (9), we have

$$
\int_{\sigma} f_{r} d H^{n-1} \geqq C\left[V, C_{0}, C_{1}\right] \quad \text { for } r \leqq r_{0} .
$$

In light of the fact that $\left\|f_{r}-|\nabla v|^{n-1}\right\|_{n^{\prime}} \rightarrow 0$ as $r \rightarrow 0$ (3.6), the result follows from (iv) and (v) of 2.5 and (10).

3.9. Lemma. Let $u$ be the extremal function for $C\left[G, C_{0}, C_{1}\right]$ (see 3.4 and 2.3). Then for $M_{n}$,-a.e. $\sigma \in \Sigma$,

$$
\int_{\sigma \cap R}|\nabla u|^{n-1} d H^{n-1} \geqq C\left[G, C_{0}, C_{1}\right]=C .
$$


Proof. Let $V_{i}$ be a sequence of open connected sets such that

$$
G \supset \mathrm{cl} V_{i+1} \supset V_{i+1} \supset \mathrm{cl} V_{i} \supset V_{i} \supset C_{0} \cup C_{1}
$$

and $G=\bigcup V_{i}$. Let $v_{i}$ be the extremal function for $C\left[V_{i}, C_{0}, C_{1}\right]=C_{i}$.

We will first show that $C_{i} \rightarrow C$ as $i \rightarrow \infty$. Recall that $C<\infty$. If $i>j$, then the restriction of $v_{i}$ to $V_{j}$ is an admissible function for $V_{j}$ and thus, so is $2^{-1} \cdot\left(v_{i}+v_{j}\right)$. As in the proof of 2.5.1, an application of Clarkson's inequality [C] gives

$$
\int_{R}\left|\frac{\nabla v_{i}-\nabla v_{j}}{2}\right|^{n} d L_{n} \leqq \frac{1}{2} C_{i}-\frac{1}{2} C_{j} \quad \text { for } i>j .
$$

Since $C_{i}$ is a monotonically increasing sequence bounded above by $C,(11)$ implies the existence of a vector-valued function $f$ such that

$$
\int_{R}\left|\nabla v_{i}-f\right|^{n} d L_{n} \rightarrow 0 \quad \text { as } i \rightarrow \infty
$$

In fact, since $C_{0} \cup C_{1}$ consists only of a finite number of nondegenerate continua, an argument similar to that of [G2, p. 362] shows that there is an admissible function $u^{\prime}$ such that $\nabla u^{\prime}=f L_{n}$-a.e. on $R$. Thus, (12) shows that

$$
\lim _{i \rightarrow \infty} C_{i}=C \text {. }
$$

This also implies that $u^{\prime}$ is the extremal function for $C$, i.e., $u^{\prime}=u$.

Since $\left\|\left|\nabla v_{i}\right|-|\nabla u|\right\|_{n} \rightarrow 0$ as $i \rightarrow \infty$, there is a subsequence of $\left|\nabla v_{i}\right|$ (which will still be denoted by $\left.\left|\nabla v_{i}\right|\right)$ such that $\left|\nabla v_{i}\right| \rightarrow|\nabla u| L_{n}$-a.e. and therefore that $\left|\nabla v_{i}\right|^{n-1} \rightarrow|\nabla u|^{n-1} L_{n}$-a.e. on $R$. This fact, along with

$$
\left\|\left|\nabla v_{i}\right|^{n-1}\right\|_{n^{\prime}} \rightarrow\left\||\nabla u|^{n-1}\right\|_{n^{\prime}}
$$

leads to

$$
\left\|\left|\nabla v_{i}\right|^{n-1}-|\nabla u|^{n-1}\right\|^{n^{\prime}} \rightarrow 0 \quad \text { as } i \rightarrow \infty .
$$

Thus, with (iv) and (v) of 2.5, we have (for another subsequence)

$$
\lim _{i \rightarrow \infty} \int_{\sigma \cap R}\left|\nabla v_{i}\right|^{n-1} d H^{n-1}=\int_{\sigma \cap R}|\nabla u|^{n-1} d H^{n-1} \quad \text { for } M_{n^{\prime}} \text {-a.e. } \sigma \in \Sigma .
$$

Lemma 3.8 states that for each $i$,

$$
\int_{\sigma \cap R}\left|\nabla v_{i}\right|^{n-1} d H^{n-1} \geqq C_{i} \quad \text { for } M_{n^{\prime}} \text {-a.e. } \sigma \in \Sigma,
$$

and therefore, the result follows from (13), (14), and (ii) of 2.5.

3.10. THEOREM. If $G$ is a bounded, open, connected set, if $C_{0} \cup C_{1}$ consists of only a finite number of nondegenerate continua, and if $C_{0} \cup C_{1} \subset G$, then

$$
M_{n^{\prime}}(\Sigma)=C\left[G, C_{0}, C_{1}\right]^{-1 / n-1} .
$$


Proof. If it were the case that $H^{n-1}$ (bdry $\left.G\right)<\infty$, then the result would follow immediately from 3.3 and 3.9. For, if we let $f=|\nabla u|^{n-1} \cdot C^{-1}$, where $u$ is the extremal for $C$, then by 3.9 there is $\Sigma_{0} \subset \Sigma$ such that $f \wedge \Sigma_{0}$ and $M_{n^{\prime}}\left(\Sigma_{0}\right)=M_{n^{\prime}}(\Sigma)$. Hence, by 3.3 ,

$$
C^{-1 / n-1} \leqq M_{n^{\prime}}(\Sigma) \leqq \int_{R} f^{n^{\prime}} d L_{n}=C^{-1 / n-1}
$$

In order to eliminate the assumption $H^{n-1}$ (bdry $\left.G\right)<\infty$, select a sequence of open, connected sets $V_{1} \subset V_{2} \subset \ldots$ such that $C_{0} \cup C_{1} \subset V_{1}, H^{n-1}\left(\right.$ bdry $\left.V_{i}\right)<\infty$, and $G=\bigcup V_{i}$. As in the proof of 3.9, let $v_{i}$ be the extremal for $C\left[V_{i}, C_{0}, C_{1}\right]=C_{i}$ and again we have

$$
C_{i} \rightarrow C,\left\|\left|\nabla v_{i}\right|^{n-1}-|\nabla u|^{n-1}\right\|_{n^{\prime}} \rightarrow 0 \quad \text { as } i \rightarrow \infty .
$$

Thus, for a subsequence

$$
\lim _{i \rightarrow \infty} \int_{\sigma \cap R}\left|\nabla v_{i}\right|^{n-1} d H^{n-1}=\int_{\sigma \cap R}|\nabla u|^{n-1} d H^{n-1} \quad \text { for } M_{n^{\prime}} \text {-a.e. } \sigma \in \Sigma .
$$

For each $i$, every $\sigma \in \Sigma$ separates $C_{0}$ from $C_{1}$ in $V_{i}$, and thus applying 3.9 with $V_{i}$ replacing $G$, we have

$$
\int_{\sigma \cap R}\left|\nabla v_{i}\right|^{n-1} d H^{n-1} \geqq \int_{\sigma \cap V_{i}}\left|\nabla v_{i}\right|^{n-1} d H^{n-1} \geqq C_{i} \quad \text { for } M_{n^{\prime}} \text {-a.e. } \sigma \in \Sigma .
$$

(Observe that 3.9, with $V_{i}$ replacing $G$, applies only to $\Sigma_{i}$, which are those sets that separate $C_{0}$ from $C_{1}$ in $V_{i}$. However, a class in $\Sigma$ which is $M_{n^{\prime}}$-zero relative to $\Sigma_{i}$ is $M_{n}$-zero relative to $\Sigma$.) Hence, in view of (15) and (16),

$$
\int_{\sigma \cap R}|\nabla u|^{n-1} d H^{n-1} \geqq C \quad \text { for } M_{n^{\prime} \text {-a.e. } \sigma \in \Sigma}
$$

which, as we have seen from above, is sufficient to establish the theorem.

3.11. Corollary. With the hypotheses of 3.10, and if $u$ is the extremal for $C\left[G, C_{0}, C_{1}\right]$, then

(i) $0 \leqq u(x) \leqq 1$ for all $x \in G$,

(ii) $\int_{\sigma \cap R}|\nabla u|^{n-1} d H^{n-1} \geqq C\left[G, C_{0}, C_{1}\right]$ for $M_{n^{\prime}}$-a.e. $\sigma \in \Sigma$,

(iii) $\int_{u^{-1}(s)}|\nabla u|^{n-1} d H^{n-1}=C\left[G, C_{0}, C_{1}\right]$, for $L_{1}$-a.e. $s \in[0,1]$.

Proof. By truncating $u$ at levels 1 and 0 if necessary, a new admissible function would be formed whose gradient would be bounded above by the gradient of $u$. However, the extremal is unique and thus (i) follows. (ii) is just a restatement of (17).

In order to prove (iii), let

$$
F(s)=\int_{u^{-1}(s)}|\nabla u|^{n-1} d H^{n-1} \quad \text { for } L_{1} \text {-a.e. } s,
$$


and observe that since $G$ is connected, $u^{-1}(s) \in \Sigma$ whenever $0<s<1$. Thus, (ii) above and 3.2 imply that $F(s) \geqq C$ for $L_{1}$-a.e. $s \in[0,1]$. However, (i) and an application of 2.2.1 give

$$
C=\int_{R}|\nabla u|^{n} d L_{n}=\int_{0}^{1} F(s) d L_{1}(s)
$$

which implies that $F(s)=C$ for $L_{1}$-a.e. $s \in[0,1]$.

3.12. REMARK. The following observation has some interest in view of Theorem 2 of [G2].

In addition to the hypotheses of 3.11 , assume that $H^{n-1}\left(C_{0}\right)=0$. Then there is a point $x_{0} \in C_{0}$ such that

$$
\limsup _{x \rightarrow x_{0}}|\nabla u(x)|=\infty .
$$

For if this were not the case, then, since $C_{0}$ is compact, there would be a constant $K>0$ and an open set $U$ such that $G-C_{1} \supset \operatorname{cl} U \supset U \supset C_{0}$ and $|\nabla u|^{n-1}<K L_{n}$-a.e. on $U$. Choose $\varepsilon>0$. Since $H^{n-1}\left(C_{0}\right)=0, C_{0}$ can be covered by a countable number of open $n$-balls $B_{i}$ such that

$$
\bigcup B_{i} \subset U \text { and } \sum H^{n-1}\left(\text { bdry } B_{i}\right)<\varepsilon K^{-1} \text {. }
$$

Since $C_{0}$ is compact, a finite number of the $B_{i}$ will cover $C_{0}$, say $B_{1}, B_{2}, \ldots, B_{k}$. According to (vi) of 2.5 , there is a nonnegative Borel function $f \in \mathscr{L}^{n^{\prime}}(R)$ such that (ii) of 3.11 fails to hold for only those $\sigma \in \Sigma$ for which

$$
\int_{\sigma \cap R} f d H^{n-1}=\infty \text {. }
$$

By employing 2.2.1, we can replace each $n$-ball $B_{i}, i=1,2, \ldots, k$, by a slightly larger one $B_{i}^{\prime}$ such that

$$
\int_{S_{i}^{\prime}} f d H^{n-1}<\infty \quad \text { where } S_{i}^{\prime}=\text { bdry } B_{i}^{\prime},
$$

$|\nabla u|^{n-1}<K H^{n-1}$-a.e. on $S_{i}^{\prime}$, and (18) still holds. Now let $\sigma=$ bdry $\left(\bigcup B_{i}^{\prime}\right)$. Then $\sigma \in \Sigma$ and (ii) of 3.11 implies that $C\left[G, C_{0}, C_{1}\right]<\varepsilon$, which means that it is zero since $\varepsilon$ is arbitrary. This would mean that $\nabla u=0 L_{n}$-a.e. on $G$. That is, since $G$ is connected and $u$ is ACT on $G, u$ would be constant, a contradiction.

In the following theorem, we will consider the general case of two disjoint compact sets $C_{0}$ and $C_{1}$ which are contained in the closure of an open, bounded, connected set $G$.

3.13. THEOREM. $M_{n} \cdot(\Sigma)=C\left[G, C_{0}, C_{1}\right]^{-1 / n-1}$.

Proof. In view of 3.3, we may assume that $C=C\left[G, C_{0}, C_{1}\right] \neq 0$. For each positive integer $i$ let

$$
\begin{aligned}
& K_{0}(i)=\operatorname{cl}\left\{x: \delta\left(x, C_{0}\right)<(2 i)^{-1}\right\} \text { and } \\
& H_{0}(i)=\left\{x: \delta\left(x, C_{0}\right)<i^{-1}\right\} .
\end{aligned}
$$


Define $K_{1}(i)$ and $H_{1}(i)$ similarly and let $G_{i}=G \cup H_{0}(i) \cup H_{1}(i)$. Since $G$ is connected, it is clear that $G_{i}$ is open and connected and notice that $K_{0}(i) \cup K_{1}(i)$ consists only of a finite number of nondegenerate continua. We will consider only those $i$ for which $K_{0}(i)$ and $K_{1}(i)$ are disjoint. Thus, let $v_{i}$ be the extremal function for $C_{i}=C\left[G_{i}, K_{0}(i), K_{1}(i)\right]$ and observe that if $i>j$, then the restriction of $v_{j}$ to $G_{i}$ is an admissible function for $C_{i}$. Finally, let $\Sigma_{i}$ be those sets $\sigma$ that separate $K_{0}(i)$ from $K_{1}(i)$ in $G_{i}$ and subject to the condition that $\sigma \cap\left[H_{0}(i) \cup H_{1}(i)\right]=0$. The purpose for this requirement is that now an $M_{n^{\prime}}$-null class in $\Sigma_{i}$ is also $M_{n^{\prime}}$ null in $\Sigma$. It is clear that $\Sigma_{1} \subset \Sigma_{2} \subset \ldots, \Sigma_{i} \subset \Sigma$ for all $i$, and

$$
\bigcup_{i=1}^{\infty} \Sigma_{i}=\Sigma
$$

Since $C_{i}$ is a monotonically decreasing sequence bounded below by $C$, we can employ again part of the argument of 3.9 to find a vector-valued function $f$ such that

$$
\lim _{i \rightarrow \infty} \int_{E^{n}}\left|\nabla v_{i}-f\right|^{n} d L_{n}=0
$$

and therefore, for a subsequence,

$$
\lim _{i \rightarrow \infty}\left\|\left|\nabla v_{i}\right|^{n-1}-|f|^{n-1}\right\|_{n^{\prime}}=0 .
$$

Hence, if $L=\lim _{i \rightarrow \infty} C_{i}, g_{i}=\left|\nabla v_{i}\right|^{n-1} \cdot C_{i}^{-1}$, and $g=|f|^{n-1} \cdot L^{-1}$ then (v) of 2.5 provides another subsequence such that

$$
\lim _{i \rightarrow \infty} \int_{\sigma \cap R} g_{i} d H^{n-1}=\int_{\sigma \cap R} g d H^{n-1} \quad \text { for } M_{n} \text {-a.e. } \sigma \in \Sigma .
$$

Now by employing 3.11 with $G, C_{0}, C_{1}$ replaced by $G_{i}, K_{0}(i), K_{1}(i)$ respectively, we have for each $i$,

$$
\int_{\sigma \cap R} g_{i} d H^{n-1} \geqq 1 \quad \text { for } M_{n^{\prime}} \text {-a.e. } \sigma \in \Sigma_{i} .
$$

Therefore, (ii) of 2.5, (19), and (21) imply

$$
\int_{\sigma \cap R} g d H^{n-1} \geqq 1 \quad \text { for } M_{n^{\prime} \text {-a.e. } \sigma \in \Sigma} \text {. }
$$

Since $v_{i}$ is the extremal for $C_{i}$, (ii) of 3.11 and 3.3 show that, for each $i$,

$$
\int_{E^{n}}\left(g_{i}\right)^{n^{\prime}} d L_{n}=C_{i}^{-1 / n-1} .
$$

Thus, with (20), (22), and (23), we have

$$
C^{-1 / n-1} \geqq \lim _{i \rightarrow \infty} C_{i}^{-1 / n-1}=\lim _{i \rightarrow \infty} \int_{E^{n}}\left(g_{i}\right)^{n^{\prime}} d L_{n}=\int_{E^{n}} g^{n^{\prime}} d L_{n} \geqq M(\Sigma) .
$$

Theorem 3.3 gives the opposite inequality, and thus the proof is complete.

We will conclude with a result concerning null sets for conformal capacity. 
3.14. TheOREM. Suppose $C_{0}$ and $C_{1}$ are disjoint compact sets in the closure of G. If $E \subset G-\left(C_{0} \cup C_{1}\right)$ is a compact set with $H^{n-1}(E)=0$, then

$$
C\left[G, C_{0}, C_{1}\right]=C\left[G-E, C_{0}, C_{1}\right] .
$$

Proof. The topological dimension of $E$ is no more than $n-2$ since $H^{n-1}(E)=0$ and therefore, $G-E$ is connected. Thus, the right hand side of the equality has meaning. Clearly,

$$
C\left[G, C_{0}, C_{1}\right] \geqq C\left[G-E, C_{0}, C_{1}\right] .
$$

The opposite inequality will be established by considering separating sets. Let $\Sigma^{*}$ be those sets that separate $C_{0}$ from $C_{1}$ in

$$
\left[(G-E)-\left(C_{0} \cup C_{1}\right)\right] \cup\left[C_{0} \cup C_{1}\right]
$$

and let $\Sigma$ be those that separate $C_{0}$ from $C_{1}$ in $R^{*}$. In light of (24) and 3.13 it is sufficient to prove

$$
M_{n^{\prime}}(\Sigma) \geqq M_{n^{\prime}}\left(\Sigma^{*}\right) .
$$

To this end, let $f$ be a function for which $f \wedge \Sigma$. In order to establish (25), we need only show $f \wedge \Sigma^{*}$. Choose $\sigma^{*} \in \Sigma^{*}$ and notice that $\sigma^{*} \cup E \in \Sigma$. Thus,

$$
\int_{\sigma^{*} \cup E} f d H^{n-1} \geqq 1
$$

and since $H^{n-1}(E)=0$,

$$
\int_{\sigma^{*}} f d H^{n-1} \geqq 1
$$

This shows that $f \wedge \Sigma^{*}$ and consequently, proves the theorem.

If $G$ is compactified $E^{n}$, Bagby has shown that $C\left[G, C_{0}, C_{1}\right]=M_{n}(\Gamma)$ where $\Gamma$ is the family of all arcs that meet both $C_{0}$ and $C_{1}$ (Ph.D. thesis, Harvard Univ., Cambridge, Mass., 1966). By using 3.13, [FU], and [W] one can show that this result is valid when $G$ is an open, bounded, connected set and $C_{0} \cup C_{1} \subset G$. (Moreover, if $C_{0} \cup C_{1} \subset \mathrm{cl} G$, the result is also valid if certain conditions are imposed on the tangential behavior of (bdry $G) \cap\left(C_{0} \cup C_{1}\right)$.) Thus, if $\Gamma^{*}$ is the family of arcs that join $C_{0}$ to $C_{1}$ in $G-E$, then 3.14 implies

$$
M_{n}\left(\Gamma^{*}\right)=M_{n}(\Gamma)
$$

This result was obtained by Väisälä [V2] in the case where $C_{0}$ and $C_{1}$ are nondegenerate continua.

\section{BIBLIOGRAPHY}

[C] J. A. Clarkson, Uniformly convex spaces, Trans. Amer. Math. Soc. 40 (1936), 396-414. [DG] E. DeGiorgi, Nuovi teoremi relativi alle misure $(r-1)$-dimensionali in uno spazio ad r dimensioni, Ricerche Mat. 4 (1955), 95-113.

[F1] F. Federer, The Gauss-Green theorem, Trans. Amer. Math. Soc. 58 (1945), 44-76. 
[F2] - The $(\varphi, K)$ rectifiable subsets of $n$ space, Trans. Amer. Math. Soc. 62 (1947), 114-192.

[F3] —, A note on the Gauss-Green theorem, Proc. Amer. Math. Soc. 9 (1958), 447-451.

[F4] - Curvature measures, Trans. Amer. Math. Soc. 93 (1959), 418-491.

[FF] H. Federer and W. H. Fleming, Normal and integral currents, Ann. of Math. 72 (1960), 458-520.

[FL] W. H. Fleming, Flat chains over a finite coefficient group, Trans. Amer. Math. Soc. 121 (1966), 160-186.

[FU] B. Fuglede, Extremal length and functional completion, Acta Math. 98 (1957), 171-219.

[G1] F. W. Gehring, Symmetrization of rings in space, Trans. Amer. Math. Soc. 101 (1961), 499-519.

[G2] - Rings and quasiconformal mappings in space, Trans. Amer. Math. Soc. 103 (1962), 353-393.

[G3] - Extremal length definitions for the conformal capacity in space, Michigan Math. J. 9 (1962), 137-150.

[H] J. Hersch, Longeurs extremales dans l'espace, resistance electrique et capacité, C. R. Acad. Sci. Paris 238 (1954), 1639-1641.

[K] V. V. Krivov, Some properties of moduli in space, Dokl. Akad. Nauk. SSSR 154 (1964), 510-513 = Soviet Math. Dokl. 5 (1964), 113-117.

[SA] B. V. Sabat, The modulus method in space, Dokl. Akad. Nauk. SSSR 130 (1960), 1210-1213 = Soviet Math. Dokl. 1 (1960), 165-168.

[SS] S. Saks, Theory of the integral, Monografje Matematyczne, Warsaw, 1937.

[S] K. T. Smith, A generalization of an inequality of Hardy and Littlewood, Canad. J. Math. 8 (1956), 157-170.

[V1] J. Väisälä, On quasiconformal mapping in space, Ann. Acad. Sci. Fenn. Ser. AI 296 (1961), 1-36.

[V2] - On the null-sets for extremal distances, Ann. Acad. Sci. Fenn. Ser. AI 322 (1962), 1-12.

[W] $\mathrm{H}$. Wallin, A connection between $\alpha$-capacity and $L^{p}$-classes of differentiable functions, Ark. Mat. 5 (1963-1965), 331-341.

[Z1] W. P. Ziemer, Integral currents mod 2, Trans. Amer. Math. Soc. 105 (1962), 496-524.

[Z2] — , Some lower bounds for Lebesgue area, Pacific J. Math. 19 (1966), 381-390.

INDIANA UNIVERSITY,

BLOOMINGTON, INDIANA 